\title{
The ACT Hand: Design of the Skeletal Structure
}

\author{
Michael Vande Weghe ${ }^{1}$, Matthew Rogers ${ }^{1}$, Michael Weissert ${ }^{2}$, Yoky Matsuoka ${ }^{1,2}$ \\ ${ }^{1}$ Robotics Institute, ${ }^{2}$ Mechanical Engineering \\ Carnegie Mellon University \\ Pittsburgh, PA, USA
}

\begin{abstract}
Robotic hands built for manipulation are often anthropomorphic but not anatomically accurate. We are constructing an anatomically-correct testbed (ACT) of the human hand to understand its mechanisms, function, and control. We have previously demonstrated that an accurate model of the extensor mechanism in the ACT Hand is crucial in realizing the human-like finger movements. Here, we present design of the bones and joints that form the skeletal structure for the ACT Hand. The bones are machined from human bone data, and are accurate in surface shape, mass, and center-of-gravity, while joints have been designed to match both degrees-of-freedom and passive stiffness. Our evaluation of the assembled index finger confirms the anatomic properties, and reveals the function of some of the peculiar shapes of the finger bones, necessity of matching the joint passive stiffness properties, and connections of the extensor mechanism.
\end{abstract}

Keywords-Humanoid Robots; Biologically Inspired Systems, Biomechanics, Hand, Hand Surgery, Anatomically-Correct Testbed

\section{INTRODUCTION}

We are building an anatomically-correct testbed (ACT) hand to serve in three capacities:

- As a telemanipulator that mimics both the active and passive dynamics of a human hand for precision teleoperation and prosthetics.

- As an experimental testbed to investigate the complex neural control of human hand movements,

- As a working physical model of the human hand for neuro- and plastic-surgeons to test new surgical reconstruction techniques for impaired hands, and

Although there exists a variety of anthropomorphic or biologically-inspired robotic hands, none of them are suitable for the above uses since they are not anatomically-correct. Robotic hands are often built with fewer degrees of freedom $[1,2,3,4]$ in order to approximate the grasping ability of the hand while simplifying the joint mechanisms. Even fingers designed with the anatomically-accurate four degrees of freedom [5,6,7] often have a different number of actuators or joint axes that don't match their human counterparts. The medical community is a rich source of static models of particular systems, such as bones, muscles, and tendons, but has not developed working prototypes that incorporate all the mechanical components into a single working model.

Our approach to building an ACT hand is to duplicate any of the features and properties which affect the static and dynamic performance of the human hand. Thus, we seek to use

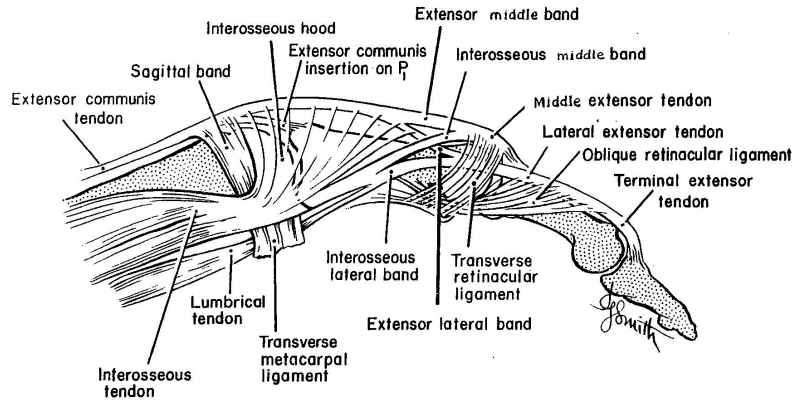

Figure 1: The extensor mechanism of the human finger (from [9])

materials that are similar in strength, mass, and elasticity to mimic the scale and geometry of the various components, and to activate and control the model in a biologically-motivated way. We do not, however, intend to duplicate features or properties that do not have a direct impact on the hand's physical performance. We have begun by building an anatomically-correct model of the index finger, which we will later use as a basis for building an entire hand.

Much of our effort to date has gone into developing a working model of the index finger's extensor mechanism. The extensor mechanism (shown in Fig. 1) is the name given to the complex web of tendons located on the dorsal side of each finger that assists in extending it and also moving it from side to side (abduction/adduction). Our version of the extensor mechanism, as described in [8], is fabricated from a composite of woven nylon and synthetic rubber, and is intended to duplicate the geometry and functionality of the human extensor mechanism. We identified that this extensor mechanism gives independent control of the metacarpophalangeal (MCP) joint and acts not only as an extensor but also as a flexor, abductor, adductor, or rotator depending on the finger's posture.

As we evaluated the extensor mechanism, we determined that bones and joints must mimic the human form closely to preserve the human dynamic behavior. In this paper we describe the design and fabrication of the anatomically correct bones and joints for our index finger model that allow the simulation of the human dynamic behavior. We first present our techniques for mimicking the size, shape, strength, and mass properties of human bones. Next, we describe our engineered joints that constrain the finger to the correct geometry while matching the low-friction properties of human joints filled with synovial fluid. We continue by outlining the steps taken to fabricate the components, and conclude with an evaluation of our results. The scope of this paper does not 




Figure 2: Datum curves in Pro/Engineer as extracted from the STL file

include our proposed actuation system, which encompasses the motors, sensors, and controllers that substitute for the human neuro-muscular system (see [10]).

\section{BONE DESIGN}

An early version of our finger model described in [8] was built with simplified bone shapes, with each bone represented by a cylinder of uniform diameter. We assumed that the shape of the bones would have little effect on the overall behavior of the finger as long as we preserved the properties of the extensor mechanism. Additionally, we hollowed out each cylinder in order to reduce the overall weight, but we did not attempt to precisely match the mass properties of human bones.

We soon discovered that the performance of the extensor mechanism was very sensitive to the shape of the underlying bones it was in contact with. Our cylindrical bones provided only a line of contact with the taught extensor mechanism, as opposed to the broad contact that human bones would afford. The geometric bones also produced a constant radius of curvature of the extensor mechanism about each joint, resulting in an effective moment arm that remained constant as the joint rotated, as opposed to the variable moment arms reported in [11]. Furthermore, the mass and the first order moment (center-of-mass) of our bones did not match their human counterparts, which affects the dynamic performance of the finger.

Our current design corrects these shortcomings by accurately matching the size, shape, and mass properties of human bones. We used 3-dimensional human bone data to model all of the subtle bumps, dips, and curves in our CAD system (Pro/Engineer) and integrated our mechanical joints directly into our bone design. Importing data from the Visible Human archive [12] (by digitizing the bone outlines from the frames of MRI data and from the photographic slice images) did not yield our target spatial resolution of $0.5 \mathrm{~mm}$ between adjacent points. Laser scanning of cadaver bones would have yielded satisfactory results, but was not financially viable. As a solution we used an existing laser-scan model of human lefthand bones from Stratasys Corporation in STL format with a nominal spatial resolution of $0.25 \mathrm{~mm}$.

The STL model was superb at modeling the bone bumps, dips, and curves, but was not directly transferable to our CAD software because the STL format represents shapes as a set of

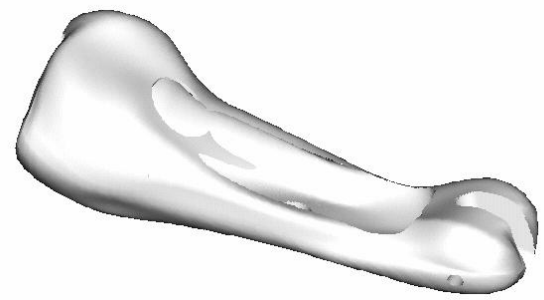

Figure 3: The cutout on the palmer side of the proximal phalanx reduces the overall mass in order to match its human counterpart.

triangular-faceted surfaces instead of solid geometry. To convert the data from the STL model to a solid model, we wrote a $\mathrm{C}++$ program to extract the facet edges and join them into continuous curves, which were then imported into Pro/Engineer (Fig. 2). Using these curves we fit surfaces to the curves and created a solid model from the closed surfaces. The final result was a solid model that could be modified in Pro/Engineer to add our additional mechanical features.

Once we correctly modeled the bone surface shapes we investigated the mass and first order moment of the ACT Hand bones and joints. We calculated the human bone properties by assigning our models a density of $1.9 \mathrm{~g} / \mathrm{cm}^{3}$ (human bone, from [13]). In addition, small cylindrical volumes were added to represent the estimated synovial fluid at the proximal end (assigned a density equal to that of water, $1.0 \mathrm{~g} / \mathrm{cm}^{3}$ ) to mimic the joint mass properties. The calculated mass properties (reported in the first column of Table 1) were used as our target values for the engineered bone and joint models.

In developing the ACT hand we modified the human bone models to accommodate our mechanical joints and the attachment points for the tendons. Since our planned materials had densities higher than that of human bone and joints $\left(2.8 \mathrm{~g} / \mathrm{cm}^{3}\right.$ for 7075 aluminum and $7.9 \mathrm{~g} / \mathrm{cm}^{3}$ for steel) the overall mass of the bones and the joints had to be reduced without affecting their interaction with the other parts of the finger. The extensor mechanism contacts the dorsal side of the finger bones throughout their range of motion, so the surfaces of the dorsal sides of the bones were not changed. Instead, we created cavities on the palmer side of each bone, where rigid tendon sheaths secure the tendons in position without relying on the shape of the underlying bone. To maintain machinability these cavities were fully exposed to the part surface (since fully- or partially-enclosed cavities cannot be easily milled). Due to the irregular surfaces, the shapes and locations of these fully exposed cavities were optimized iteratively. We terminated when the bone mass matched within $5 \%$ and the deviation between the two centers-of-mass was less than $1.5 \%$ of the bone's overall length, yielding the results shown in Table 1.

The proximal and middle phalanges required only a few iterations to achieve the results, but the distal bone was so small that our termination condition could not be reached. Instead, we removed as much material as we could without sacrificing strength to arrive at a design that was less than $0.15 \mathrm{~g}$ heavier than the human bone. The final proximal phalanx with its palmer side cutout is shown in Fig. 3 (the cutouts on the mid and distal phalanges have similar shapes). 
TABLE I. COMPARISON OF MASS PROPERTIES FOR SAMPLE HUMAN FINGER BONE VERSUS ENGINEERED ALUMINUM-STEEL MODEL

\begin{tabular}{|c|l|l|l|l|l|}
\hline Phalanx & $\begin{array}{c}\text { Human } \\
\text { Mass } \\
(\mathrm{g})\end{array}$ & $\begin{array}{c}\text { ACT } \\
\text { Mass } \\
(\mathrm{g})\end{array}$ & $\begin{array}{c}\text { ACT/Human } \\
\text { Mass ratio } \\
(\%)\end{array}$ & $\begin{array}{c}\text { COM } \\
\text { Deviation } \\
(\mathrm{mm})\end{array}$ & $\begin{array}{c}\text { COM } \\
\text { Deviation } \\
\text { Ratio } \\
(\%)\end{array}$ \\
\hline Proximal & 12.4 & 11.9 & $96 \%$ & 0.48 & $0.82 \%$ \\
\hline Middle & 3.6 & 3.5 & $96 \%$ & 0.42 & $1.3 \%$ \\
\hline Distal & 0.97 & 1.1 & $114 \%$ & 0.83 & $3.4 \%$ \\
\hline
\end{tabular}

We did not match the mass of the metacarpal bone since in our ACT Hand configuration the metacarpal is stationary and thus its mass does not affect the dynamic performance of the finger.

\section{JOINT DESIGN}

The joint properties in the human finger play an equally important roll in the finger's performance. In order for our model finger to behave identically to the human finger, we matched three critical properties at each joint. First, the geometry of each joint was matched to have the same degrees of freedom, range of motion, and relationships between the axes as its human counterpart. We also mimicked the internal friction of each joint. Finally, the elastic properties of each joint were conserved to ensure consistent dynamic performance of each joint by itself and in relation to the other joints. Most importantly, these critical properties had to be designed without affecting the other components of the system.

Although the human finger joints resemble ball-and-socket joints in appearance, the curved, mating layers of cartilage have enough contours built into them that the degrees of freedom are reduced. The metacarpophalangeal (MCP) joint has only two degrees of freedom, while the proximal inter-phalangeal (PIP) and distal inter-phalangeal (DIP) joints move like almost perfect single-axis rotational joints. Although it is true that the degrees-of-freedom for each joint could be duplicated by copying the shape of the cartilage on each bone and constructing the appropriate ligaments to hold the joints together, we would not be able to match the low friction since we do not have an appropriate substitute for the synovial fluid that lubricates each joint. The problem is compounded during motions that require co-contraction of opposing muscles, since the effect is to increase the normal force on the mating joint surfaces, thus driving the frictional forces even higher. Our solution was to implement the joints in our model using traditional engineered joints fabricated from lubricated metal bushings and ball bearings. In this manner the friction was kept low without having to retain lubricant as in a fluid-based joint.

We designed the PIP and DIP joints based on a steel link arm rotating about a steel axle. The use of the small-diameter link arm allowed us to keep the geometry of the bones unchanged, so that one bone end glides past its neighbor with only a small gap in between (the gap that in the human joint is filled with cartilage and synovial fluid). The only visible modification to the bones was the slot cut in the distal ends of the bones to allow for the movement of the link arm.

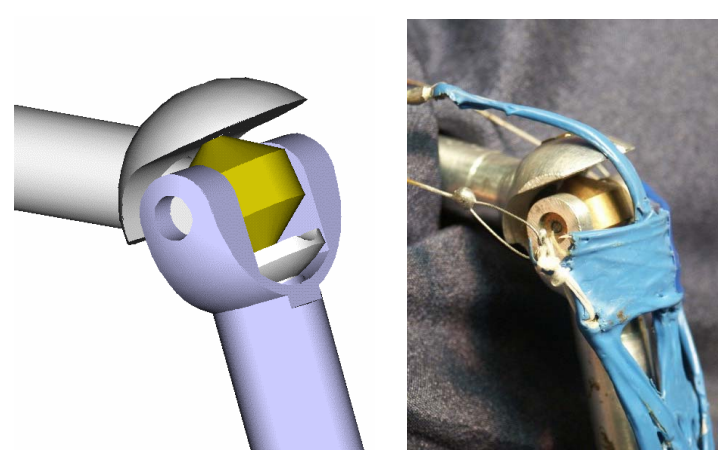

Figure 4: The previous MCP joint with its large exposed gimbal.

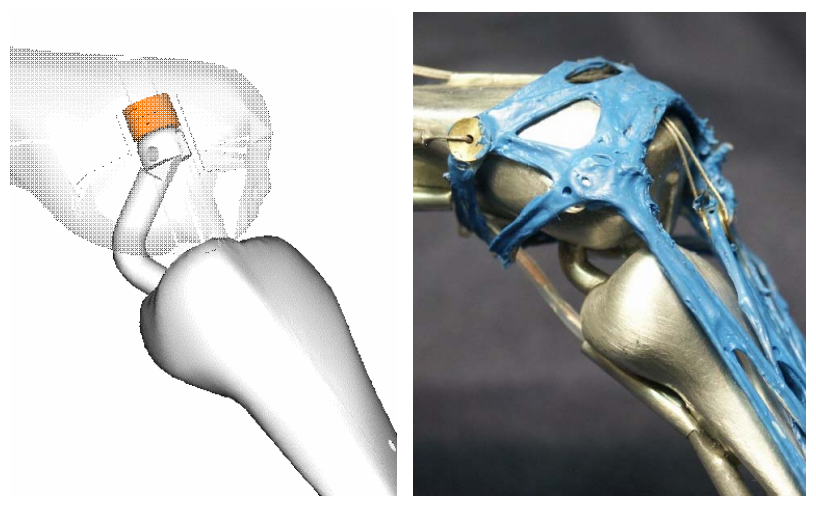

Figure 5 The revised MCP joint, consisting of a miniature internal gimbal mechanism and an angled linkarm

Rotational limits for each of the joints were imposed by the internal shape of the slot. Encapsulating ligaments that are required to hold together the human joints were not employed since the mechanical joints already constrain the motion to only a single degree of freedom.

The mechanism we employed for the MCP joint was more complex than the PIP/DIP joint design to allow for abduction/adduction motions. Our enabling technology was to use a gimbal mechanism that incorporated two coupled degrees of rotational freedom. By tilting the primary axis back from vertical by 30 degrees as modeled in [14] a small amount of axial rotation in the extended finger was created as it was abducted/adducted, and restricted the abduction/adduction when the finger was flexed by 60 degrees, just as in the human finger.

For the MCP joint, a traditional larger gimbal used in our previous design [8] shown in Fig. 4 did not preserve the anatomical features. To accommodate the motion of the proximal phalanx connections, a large opening was made in the distal end of the metacarpal bone. The edge of this opening created a lip that caught the extensor mechanism as the finger flexed. Furthermore, the cavity left nothing to support the extensor mechanism when the interosseus muscles were activated while the finger was flexed, causing the extensor mechanism to be pulled deep into the cavity.

Our design used a miniature version of the gimbal mechanism with a narrow link arm connecting the second axis to the proximal phalanx as shown in Fig. 5. The gimbal was 


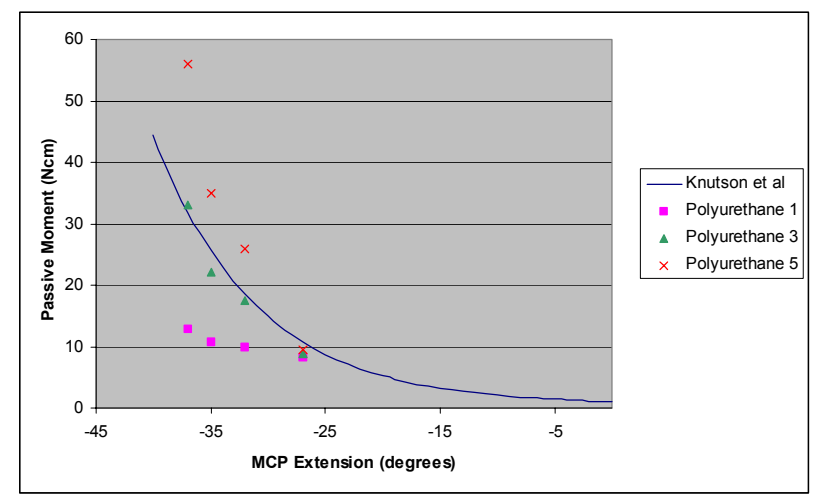

Figure 6 Comparison of MCP passive moment from [15] versus the measured passive moment created in the ACT Hand by adding pieces of three different foam compositions to the joint cavities.

supported by a pair of ball bearings for rotation about its primary axis, and incorporates a small steel axle for the link arm to rotate about the secondary axis. The 90 degree bend in the link arm permitted us to keep its movement clear of the distal end of the metacarpal, even when the finger was in full extension. Although a small cutout in the metacarpal was still required, this cavity was primarily on the palmer side of the bone where it did not interfere with the movement of the extensor mechanism. Even when the finger model was fully flexed and the distal end of the metacarpal was exposed, the cavity was barely visible.

Each muscle/tendon pair that attaches to the finger has an effect on the passive stiffness when rotated about the corresponding joint, but there also exists an internal stiffness in each joint caused in part by the structure of the ligaments that hold the joint capsule together. In [15], experiments on eight adults measured the intrinsic stiffness of the MCP joint. The data shows that typically the passive stiffness increases by approximately $25 \mathrm{Ncm}$ in the final 10 degrees of flexion and extension. We sought to match the passive stiffness by testing pieces of foam padding at each end of the joint cavity. We tested three different foam compositions by recording torque/angle data of the joint near its extension limit, and plotted the resulting values against the curve calculated in [15], as shown in Fig. 6. Based on our comparison of the curves we selected an open-cell polyurethane with a "number 3" firmness rating.

\section{FABRICATION}

Our engineered bones were machined from solid 7075-T6 aluminum rod using a 4-axis $\mathrm{CNC}$ milling machine. The tool paths were calculated directly from our CAD model, and the bones were machined in three major steps. First, the contours of the distal end and mid section of the bone were machined, and threaded attachment points were added to the palmer side of the mid section before parting off the unfinished bone from the raw stock. Second, we turned the workpiece around and mounted the bone onto a fixture using the palmer side attachment points. Gripping the workpiece in this manner allowed us to machine the proximal end of the bone. Finally, the bone mass was reduced by holding the bone palmer side up

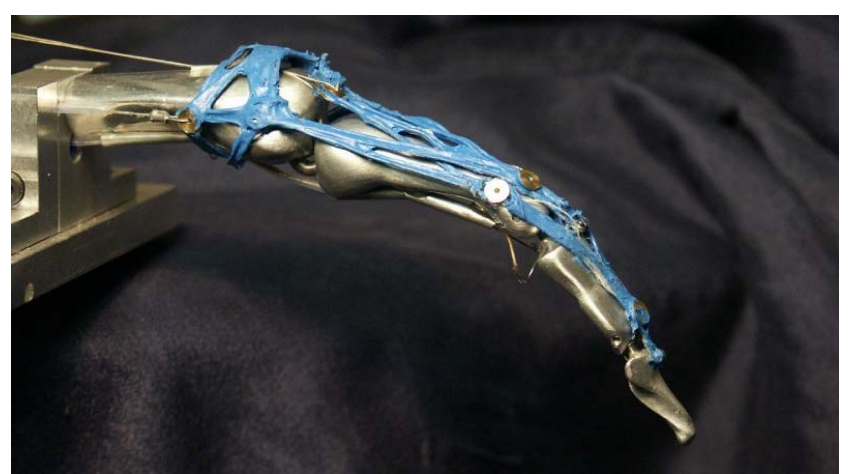

Figure 7: Assembled index finger

using low-temperature fixturing metal and cutting cavities in the palmer side using a manual 3-axis milling machine (this step also machined away our threaded attachment points). Attachment points for the extensor mechanism and the flexor tendons were added by hand tapping a series of holes.

The gimbal for the MCP joint was fabricated from 3/16" diameter 17-4 PH stainless steel rod by turning the overall shape on a CNC lathe and then adding the slot and axle hole on a manual milling machine. Link arms were also made from 17$4 \mathrm{PH}$, chosen for its high strength and ease in machining and bending. The joint axles were made by cutting hardened drill blanks to length.

Assembly of the finger model was conducted in the following order: First, the link arms were inserted into the bones and held in place with high-strength adhesive, as was the gimbal with its ball bearings. Second, axle pins were simply pressed into place so that they could be removed for maintenance. The extensor mechanism was secured to the dorsal side of the mid and distal phalanges using M1 screws passed through the brass eyelets integrated into the mechanism. Teflon tubing was used to sheath the flexor tendons, and was held in place with small lengths of aluminum tubing epoxied to the palmer side of the proximal and mid phalanges. Finally, steel cables representing the flexor tendons themselves are anchored to the mid and distal phalanges with \#1-72 set screws.

\section{EVALUATION}

Fig. 7 shows the fully assembled anatomical index finger. The smooth continuous surfaces gave adequate support to the extensor mechanism in all finger positions, and the joints allowed low-friction actuation of all of the degrees of freedom.

In evaluating the performance of the design we observed the relationship between the contours of the bones and the shape of the extensor mechanism. We found that the asymmetrical shape of the distal end of the metacarpal applies lateral pressure to the sliding central extensor connection of the extensor mechanism. Although we had previously been unable to determine a need for the sagital band connections on the extensor mechanism (refer to Fig. 1), it is clear that without the sagital bands the extensor connection would be pushed off center during flexion and tend to adduct the finger.

Additionally, the sloping sides on the top of the middle phalanx allows the conjoint lateral bands to slide away from 
each other during flexion of the PIP joint. This separation is a normal and necessary behavior in healthy fingers [16], but its extent is usually limited by the presence of the transverse retinacular ligament (in Fig. 1), which we had originally omitted from our extensor mechanism for simplicity's sake. The resulting behavior simulated the "boutonniere deformity" [17], in which a tear in the central slip of the extensor mechanism allows the conjoint lateral bands to straddle the PIP joint and pull directly on the distal phalanx. By adding a connection to the extensor mechanism to represent the transverse retinacular ligament the problem was corrected. Currently, hand surgeons attempt to splint or repair the central slip when correcting a boutonniere deformity, but our results also suggest that adding material to flatten the top of the mid phalanx might help to reduce the spreading forces and give the central slip more time to heal.

Informal evaluation of the foam padding that was added to the joint cavities shows that it improves the dynamic performance of the finger by helping it to return from positions of extreme flexion or extension. The design still requires improvement, however, due to the tendency of the foam to become dislodged during use. Our future ACT bone design will including anchoring points that will hold the foam padding in position.

As we move forward to create many more bones for other fingers and the palm, we will modify the steps we take to machine the bones. Since the palmer side cavities in our current version were made on a manual milling machine after fixturing the bone approximately in place, we did not have the accuracy required to remove all of our planned material without the risk of breaking through the part walls. In reconsidering our manufacturing steps we will $\mathrm{CNC}$ machine the palmer side cavities according to our CAD model, thereby meeting our weight and center-of-mass targets. We also plan to further evaluate our index finger to determine the crucial ligaments that needs to be added to constrain our tendons and extensor mechanism.

\section{ACKNOWLEDGMENTS}

The authors would like to thank Dr. Sean Bidic and Dr. Joseph Imbriglia for their medical insight and assistance.

\section{REFERENCES}

[1] A. Bekey, R. Tomovic, and I. Zeljkovic. Control architecture for the Belgrade/USC hand. Dextrous Robot Hands, Springer-Verlag: New York. 1990, pp. 136-149.

[2] C.S. Lovchik, and M.A. Diftler. The Robonaut Hand: a dexterous robot hand for space. Proc. IEEE Intl. Conference on Robotics and Automation, pp. 907-912, 1999.

[3] Y. Matsuoka. The mechanisms in a humanoid robot hand. Autonomous Robots, vol. 4, no. 2, pp. 199 - 209, 1997.

[4] J.K. Salisbury and J.J. Craig. Articulated hands: force control and kinematic issues. IJRR, vol. 1, no. 1, 1982.

[5] Dexterous Hand/Arm. Shadow Robot Company. http://www.shadow. org.uk/products/newhand.shtml.

[6] S.C. Jacobsen, E.K. Iversen, D.F. Knutti, and R.T. Johnson. Design of the Utah/MIT dextrous hand. Proc. IEEE Intl. Conference on Robotics and Automation, pp. 96-102, 1986.
[7] Y.K. Lee and I. Shimoyama. A skeletal framework artificial hand actuated by pneumatic artificial muscles. Proc. IEEE Intl. Conference on Robotics and Automation, pp. 926-931, 1999.

[8] D. Wilkinson, M. Vande Weghe, and Y. Matsuoka. An extensor mechanism for an anatomical robotic hand. Proc. IEEE Intl. Conference on Robotics and Automation, pp. 238-243, 2003.

[9] R. Tubiana. Architecture and functions of the hand. In R. Tubiana (ed): The Hand, vol 1. Philadelphia. WB Saunders, 1981, p. 55.

[10] N. Gialias and Y. Matsuoka. Muscle actuator design for the ACT Hand Proc. IEEE Intl. Conference on Robotics and Automation, 2004.

[11] K. N. An, Y. Ueba, E. Y. Chao, W. P. Cooney, and R. L. Linscheid. Tendon excursion and moment arm of index finger muscles. Journal of Biomechanics, vol 16, pp. 419-425, 1983.

[12] Visible Human Project, National Library of Medicine, Bethesda, MD, http://www.nlm.nih.gov/research/visible/visible_human.html.

[13] J. R. Cameron, J. G. Skofronick \& R. M. Grant. Physics of the Body. Second Edition. Madison, WI: Medical Physics Publishing, 1999, p. 96.

[14] P. Brand and A. Hollister, Clinical mechanics of the hand, $2^{\text {nd }}$ ed. St. Louis: Mosby-Year Book, Inc, 1993.

[15] J. S. Knutson, K. L. Kilgore, J. M. Mansour, and P. E. Crago. Intrinsic and extrinsic contributions to the passive moment at the metacarpophalangeal joint. Journal of Biomechanics, vol. 33, pp. 1675 $1681,2000$.

[16] C. Harris, Jr., and G. L. Rutledge, Jr. The functional anatomy of the extensor mechanism of the finger. Journal of Bone Joint Surgery [Am], vol. 54-A, pp. 713-726, 1972.

[17] W.A. Souter. The problem of boutonniere deformity. Clinical Orthopaedics, vol. 104), pp. 116-133, Oct. 1974. 